Ann. Zootech., I975, 24 (3), 4I3-422.

\title{
SUR LA MESURE DES ÉCHANGES DE CHALEUR AU NIVEAU DE LA PEAU DES PORCS ÉLEVÉS SOUS ABRI PAR LA MÉTHODE DU BILAN D'ÉNERGIE
}

\author{
P. BERBIGIER ( $\left.{ }^{1}\right)$ \\ Station de Bioclimatologie, \\ Centre de Recherches agronomiques des Antilles et de la Cinyane, I. N. R. A., \\ 97170 Petit-Bourg (Guadeloupe)
}

\section{RÉSUMÉ}

I.a méthode de mesure des échanges thermiques locaux au niveau de la peau du Porc que nous décrivons ici repose sur les principes physiques du bilan d'énergie d'une surface; la mesure de base est celle de la température superficielle de la peau, effectuée par radiothermométrie infrarouge. Les conditions de température, d'humidité, de rayonnement et éventucllement de ventiJation de l'ambiance sont aussi nécessaires, ainsi que la température rectale des porcs. Les calculs donnent les divers flux de chaleur par la peau et le coefficient de conduction du corps. Différents problèmes, souvent liés aux hypothèses physiques, sont ensuite discutés. Malgré ses imperfections, cette méthode est sans doute la seule à pouvoir être utilisée en ambiance non contrôlée.

\section{I. - IN'TRODUC'TION}

Les méthodes utilisées pour déterminer les échanges thermiques d'un animal se classent en deux catégories : d'une part la calorimétrie indirecte (ou thermochimie respiratoire) qui permet de mesurer la production de chaleur de l'animal (FULLER et Boyne, 1972; Hor,MEs, I973), d'autre part la calorimétrie directe qui permet de déterminer la dissipation de chaleur dans le milieu ambiant.

Cette mesure peut être effectuée soit globalement à l'aide d'un calorimètre (Holmes, ig68; Close, Mount et Start, I97I), soit ponctuellement à l'aide de fluxmètres (INGRAM, I964a). La deuxième méthode présente l'inconvénient d'être

(1) Adresse actuelle : Station de Bioclimatologie, Centre national de Recherches agronomiques, I. N. R. A., 78000 Versailles. 
moins précise que la première : en effet on extrapole à l'ensemble de l'animal des mesures faites localement; en revanche elle a l'avantage d'être plus facile à mettre en oeuvre.

Une variante de cette dernière méthode consiste à calculer les valeurs des échanges de chaleur au niveau de la peau à partir de la mesure des températures rectales et cutanées de l'animal et de la détermination des conditions atmosphériques ambiantes. Cette technique fait appel aux équations du bilan d'énergie. FINCH (I972) et MoRRISON (I972) proposent de plus une simplification de la mesure de la température de surface, par radiothermométrie infra-rouge. Ce procédé qui permet d'intégrer la température sur une zone étendue de la peau, fait appel à un appareillage très maniable et, la mesure s'effectuant à distance, ne perturbe pas les échanges thermiques. Il paraît particulièrement intéressant pour des études chez le Porc, animal à peau nue, dont le total des pertes cutanées (convection, conduction, radiation, évaporation) représente, si les conditions climatiques ne sont pas trop extrêmes, plus de $80 \mathrm{p}$. Ioo des pertes globales (Mount, I968). Nous avons mis au point une technique de ce type, et nous l'avons testée sur des porcelets au centre de recherches I. N. R. A. de Guadeloupe.

\section{II. - THÉORIE}

\section{A. - Équation du bilan énergétique}

En régime stationnaire, la somme algébrique des flux de chaleur échangés au niveau de la peau s'établit comme suit (KLEIBER, I96r, MORRISON, I972).

$$
\Phi_{r}+\Phi_{s}+\Phi_{l}+\Phi_{i}=\mathrm{o}
$$

$\Phi_{r}:$ flux radiatif $\left(\mathrm{W} \cdot \mathrm{m}^{-2}\right)$

$\Phi_{s}$ : flux de chaleur sensible $\left(\mathrm{W} \cdot \mathrm{m}^{-2}\right)$

$\Phi_{l}$ : flux de chaleur latente $\left(\mathrm{W} \cdot \mathrm{m}^{-2}\right)$

$\Phi_{i}$ : flux de chaleur interne $\left(\mathrm{W} \cdot \mathrm{m}^{-2}\right.$ ) (dégagée au niveau de la peau).

La règle de signe pour les flux est la suivante :

chaleur gagnée par la peau : flux positif, chaleur perdue par la peau : flux négatif.

Les différents flux s'expriment ainsi :

$$
\Phi_{r}=\varepsilon \sigma\left(\mathrm{T}_{p}^{4}-\mathrm{T}_{s}^{4}\right) \simeq \sigma\left(\mathrm{T}_{p}^{4}-\mathrm{T}_{s}^{4}\right) \simeq 4 \sigma^{\top} \mathrm{T}^{3}\left(\mathrm{~T}_{p}-\mathrm{T}_{s}\right)
$$

En effet $\varepsilon \simeq$ I (MOUNT, Ig68; cf. $\S$ III, A)

$$
\Phi_{s}=\frac{\rho c}{r_{a} \mathrm{~L}_{e^{2 / 3}}}\left(\mathrm{~T}_{a}-\mathrm{T}_{s}\right)
$$

en régime de convection forcée laminaire

$$
\begin{gathered}
\Phi_{l}=\frac{\mathrm{LM}}{\mathrm{R}_{r m} \gamma_{a}}\left(\mathrm{P}\left(\mathrm{T}_{r}\right)-\mathrm{P}\left(\mathrm{T}_{r s}\right)\right) \quad(\text { PERRIER, I975) } \\
\Phi_{i}=\mathrm{C}_{i}\left(\mathrm{~T}_{i}-\mathrm{T}_{s}\right) \quad(\text { MounT, I } 968)
\end{gathered}
$$


$\mathrm{T}_{a}$ : température de 1'air ambiant $\left({ }^{\circ} \mathrm{C}\right)$

$\mathrm{T}_{p}$ : température radiative des parois $\left({ }^{\circ} \mathrm{C}\right)$

$\mathrm{T}_{s}$ : température de surface de la peau $\left({ }^{\circ} \mathrm{C}\right)$

$\mathrm{T}_{i}$ : température rectale $\left({ }^{\circ} \mathrm{C}\right)$

$\sigma: 5,674 \times 10^{-8} \mathrm{~W} \cdot \mathrm{m}^{-2} \cdot{ }^{\circ} \mathrm{K}^{-4}$ constante de Stefan-Boltzmann.

$\varepsilon$ : émissivité de la peau

$\mathrm{T}=\left(\mathrm{T}_{s}+\mathrm{T}_{p}\right) / 2\left({ }^{\circ} \mathrm{K}\right)$

$\rho$ : masse spécifique de l'air $\left(\mathrm{kg} \cdot \mathrm{m}^{-3}\right)$

$c$ : chaleur spécifique de l'air $\left(\mathrm{J} \cdot \mathrm{kg}^{-1} \cdot \mathrm{oC}^{-1}\right)$

$r_{a}$ : résistance à la diffusion de la vapeur d'eau de la couche limite de l'air au contact de la peau $\left(\mathrm{s} \cdot \mathrm{m}^{-1}\right)$

Le : nombre de Lewis (rapport des diffusivités de la vapeur d'eau et de la chaleur)

$\mathrm{M}=\mathrm{I} 8 \times \mathrm{IO}^{-3} \mathrm{~kg} \cdot \mathrm{mole}^{-1}:$ masse molaire de l'eau

L : chaleur latente de vaporisation de l'eau $\left(\mathrm{J} \cdot \mathrm{kg}^{-1}\right)$

$\mathrm{R}=8,3 \mathrm{I} 4 \mathrm{~J} \cdot \mathrm{mole}^{-1} \cdot \mathrm{oK}^{-1}$ : constante des gaz parfaits

$\mathrm{T}_{r}$ : température de point de rosée de l'air ambiant

$\mathrm{P}\left(\mathrm{T}_{r}\right)$ : pression partielle de vapeur d'eau dans l'air $(\mathrm{P} a)$ (saturante à la température $\mathrm{T}_{r}$ )

$\mathrm{T}_{r *}$ : température de point de rosée de l'air au contact de la peau

$\mathrm{P}\left(\mathrm{T}_{r s}\right)$ : pression partielle de vapeur d'eau de l'air au contact de la peau $(\mathrm{Pa})$

$\mathrm{T}_{r m}=\frac{\mathrm{T}_{r}+\mathrm{T}_{r s}}{2}\left({ }^{\circ} \mathrm{K}\right)$

$\mathrm{C}_{i}$ : coefficient de conduction thermique du corps $\left(\mathrm{W} \cdot \mathrm{m}^{-2} \cdot \mathrm{oC}^{-1}\right)$.

Ie flux de chaleur radiative $\Phi_{r}$ peut s'exprimer ainsi dans la mesure où le rayounement de l'ambiance se limite at rayonnement thermique des parois (rayonnement visible négligeable). Dans le cas d'un porc en plein air, il faut ajouter à l'équation (I) le rayonnement visible incident sur la partie du corps considérée.

L'équation du bilan énergétique devient

$$
\begin{aligned}
4 \sigma^{\prime} \mathrm{T}^{3}\left(\mathrm{~T}_{p}-\mathrm{T}_{s}\right)+\frac{\rho c}{r_{a} \mathrm{~L}_{1}^{2 / 3}}\left(\mathrm{~T}_{a}-\mathrm{T}_{s}\right)+\frac{\mathrm{LM}}{\mathrm{RT}_{r m} r_{a}}\left(\mathrm{P}\left(\mathrm{T}_{r}\right)-\mathrm{P}\left(\mathrm{T}_{r s}\right)\right) \\
+\mathrm{C}_{i}\left(\mathrm{~T}_{i}-\mathrm{T}_{s}\right)=0
\end{aligned}
$$

Cette équation comporte trois inconnues, $r_{a}, \mathrm{C}_{i}, \mathrm{P}\left(\mathrm{T}_{r s}\right)$.

\section{B. - Détermination expérimentale de $r_{a}$}

Ce paramètre est mesuré sur des peaux mouillées et tendues sur un support isolant; on a alors saturation à la surface, et $\Phi_{i}=0$.

L'équation (6) devient

$$
4 \sigma^{\prime} \mathrm{T}^{3}\left(\mathrm{~T}_{p}-\mathrm{T}_{s}\right)+\frac{\rho c}{r_{a} \mathrm{Le}^{2 / 3}}\left(\mathrm{~T}_{a}-\mathrm{T}_{s}\right)+\frac{\mathrm{LM}}{\mathrm{RT}_{r m} \boldsymbol{T}_{a}}\left(\mathrm{P}\left(\mathrm{T}_{r}\right)-\mathrm{P}\left(\mathrm{T}_{s}\right)\right)=0
$$

où $\gamma_{a}$ est la seule inconnue. 


\section{C. - Conductivité du corps et humidité de la peau}

Pour résoudre les deux autres indéterminations, on avait imaginé de mouiller la peau de l'animal, et donc d'avoir deux valeurs de $\mathrm{T}_{s}$, sur peau sèche et sur peau humide. I a valeur sur peau humide aurait permis de lever l'indétermination sur $\mathrm{P}\left(\mathrm{T}_{r s}\right)$, car alors $\mathrm{P}\left(\mathrm{T}_{r s}\right)=\mathrm{P}\left(\mathrm{T}_{*}\right)$. On aurait donc obtenu une valeur de $\mathrm{C}_{i}$. En supposant que le mouillage ne provoque pas de modification de la circulation sanguine $\left(\mathrm{C}_{i}=\right.$ constante $)$, on aurait $\mathrm{pu}$, en reportant cette valeur dans l'équation de l'animal $\mathrm{sec}$, résoudre l'équation en $\mathrm{P}\left(\mathrm{T}_{r s}\right)$.

Malheureusement, on a constaté que si on mouille tout l'animal, on a parfois une vasoconstriction (Valeurs de $\mathrm{C}_{i}$ anormalement basses).

Par contre, si on ne mouille que la zone de mesure, les dimensions réduites des porcelets ne permettent pas d'avoir une zone mouillée suffisamment étendue pour que sa température échappe à l'influence des zones de peau sèche environnantes. On a alors une température humide trop élevée, conduisant à des estimations de $\mathrm{C}_{i}$ trop fortes et à une valeur de $\Phi_{\iota}$ aberrante sur la peau sèche (parfois 75 p. roo dela chaleur dégagée). Cette méthode a donc été abandonnée sur les porcs.

\section{D. - Approximations sur $\Phi_{l}$}

On a utilisé les valeurs trouvées par INGRAm ( $1964 a$ ) à l'aide de capsules ventilées. Fin effet, les valeurs de $\Phi_{l}$ chez le Porc sont en général très faibles (ro p. Ioo des échanges). De plus les travaux d'Ingram se sont déroulés dans des conditions de température voisines des nôtres, avec toutefois une humidité plus basse. Cependant, selon IxGRAm ( 1965$)$, on ne pourrait pas utiliser ces données : une forte humidité ambiante peut induire des flux de chaleur latente positifs. Mais Ingram a observé de tels flux après une augmentation brutale de l'humidité et pendant seulement 40 minutes ; l'équilibre des échanges par évaporation n'était sans doute pas encore atteint. Lorsqu'il l'est, l'humidité de l'air doit jouer un faible rôle sur $\Phi_{l}$ (FINDIAY, I972) : l'utilisation des données d'Ixgram semble se justifier. Les valeurs de (D sont donc de l'ordre de ro $\mathrm{W} \cdot \mathrm{m}^{-2}$ et varient avec la température de l'air ambiant.

$$
\text { E. - Conséquences sur } \Phi_{i} \text { et } C_{i}
$$

En négligeant le terme $\Phi_{l}$, si $\mathrm{T}_{p} \simeq \mathrm{T}_{a}$ (parois isolantes), on obtient, d'après l'équation (6) :

ce qui conduit à

$$
\Phi_{i} \simeq\left(\frac{\rho c}{r_{a} \mathrm{~L}_{1} \mathrm{e}^{2 / 3}}+4 \sigma^{\prime} \mathrm{T}^{3}\right)\left(\mathrm{T}_{s}-\mathrm{T}_{a}\right)
$$

$$
\mathrm{C}_{i} \simeq\left(\frac{\rho c}{r_{a} \mathrm{~L}_{1} \mathrm{e}^{2 / 3}}+4 \sigma^{\prime} \mathrm{T}^{3}\right)\left(\frac{\mathrm{T}_{8}-\mathrm{T}^{\prime}}{\mathrm{T}_{i}-\mathrm{T}_{i}}\right)
$$

On voit que $C_{i}$ est alors proportionnel à l'indice de circulation thermique $\frac{\mathrm{T}_{s}-\mathrm{T}_{n}}{\mathrm{~T}_{i}-\mathrm{T}_{s}}$ (Mount, I968). 


\section{III. - MATÉRIEL ET MESURES}

\section{A. - Radiothermomètre Heimann KT 40}

Ce modèle, conçu pour les mesures physiologiques, est utilisé pour mesurer la température de surface de la peau, ainsi que la température radiative des parois. Son principe est basé sur la. loi d'émission des corps noirs (Loi de Stefan-Bolzmann) : l'énergie radiative émise par un corps noir dans toutes les directions est proportionnelle à la puissance quatrième de sa température absolue.

La différence entre l'énergie provenant de la surface dont on cherche la température et celle provenant d'un corps de référence (maintenu à température constante) provoque une différence de potentiel qui est amplifiée et mesurée par un voltmètre incorporé à l'appareil. Ce radiothermomètre permet de mesurer, en fonctionnement normal, des températures allant de $22^{\circ} \mathrm{C}$ à $+2^{\circ} \mathrm{C}$ : sa réponse est pratiquement linéaire dans ces limites. On peut augmenter la gamme de mesure en jouant sur l'amplification. La précision de la mesure est d'environ o, $3^{\circ} \mathrm{C}$ (PERRIER, I970).

Pour les corps non noirs, caractérisés par une émissivité $\approx$ inféricure à $\mathbf{I}$, on doit appliquer une correction. Dans la gamme d'émission correspondant aux températures physiologiques $(\lambda \simeq$ Io $\mu$ ), cette émissivité est sensiblement égale à l'unité pour la peau de lá plupart des animaux ct peut être négligéc.

$$
\text { B. - Autres appareils }
$$

La température et l'humidité de l'air ambiant sont mesurées à l'aicle d'un psychromètre Assmann, la température rectale à l'aide d'un thermomètre médical sensible à o, Io C.

$$
\text { C. - Mesures }
$$

Elles consistent à déterminer, pour différentes parties lu corps de l'animal, la température de surface de la peau; au même instant, on note la température moyenne de la paroi faisant face à la région du corps considérée, la température et l'humidité de l'air ambiant, et en fin la température rectale. I'n calcul du bilan d'énergie est ensuite cffectué pour chaque point de mesure et on fait la moyenne des flux de chaleur sur l'ensemble des points. Il faut noter que, lorsque les conditions climatiques ambiantes sont sujettes à de brusques variations, il est nécessaire d'intégrer les mesures dans le temps, la théorie n'étant valable en toute rigueur qu'en régime stationnaire.

\section{IV. - DISCUSSION}

Il faut d'abord souligner que cette méthode ne rend pas compte des échanges respiratoires. Nous allons examiner les autres problèmes qu'elle pose.

\section{A. - Température de surface}

- L'émissivité $\varepsilon$ de la peau est, dans nos calculs, assimilée à l'unité. L'erreur introduite est négligeable. En effet, si la température de la peau est de $34^{\circ} \mathrm{C}$, la température des parois de $27^{\circ} \mathrm{C}$ (conditions expérimentales en climat tropical) et $\varepsilon=0,97$ (Mount, I968), l'erreur systématique introduite sur $\mathrm{T}_{s}$ par cette hypothèse sera de $-0,2^{\circ} \mathrm{C}$ (inférieure à la précision de l'appareil). Par contre, en plein air, il sera nécessaire d'introduire dans les équations la valeur réelle de $\varepsilon$ (PERRIER, I97o) ainsi que l'albédo de la peau.

- La température indiquée par le radiothermomètre est la température moyenne des surfaces qui se trouvent dans le champ de l'appareil. Dans le cas du Porc, il 
s'agit essentiellement de la peau ; pour des animaux à pelage dense, c'est la température de la surface du pelage.

- Pour savoir si la moyenne des températures de surface mesurées en quelques points du corps est une estimation satisfaisante de la température moyenne de la peau de l'animal, nous avons calculé d'une part la relation entre la valeur moyenne obtenue pour 5 points du corps (tête, dos, flanc, cuisse, épaule) et celle obtenue pour Io points (les mêmes, plus oreille, patte avant et arrière, ventre et bas-ventre), d'autre part, la relation entre la température moyenne du dos et celle des 5 premiers points.

La régression entre les moyennes de 5 points $(\mathrm{Y})$ et de ro points $(\mathrm{X})$, pour i 8 couples de valeurs, est de la forme :

$$
\mathrm{Y}=0,948 \mathrm{X}+\mathrm{I}, 68 \quad r=0,934
$$

Les valeurs de $\mathrm{Y}$ sont en général légèrement inférieures à celles de $\mathrm{X} \quad(0,2$ à $\left.0,4^{\circ} \mathrm{C}\right)$. Le coefficient de corrélation n'est pas très élevé, car la gamme de températures est étroite $\left(34^{\circ} \mathrm{C}-39^{\circ} \mathrm{C}\right)$, mais $\mathrm{X}$ et $\mathrm{Y}$ sont en général très proches.

Pour 249 couples de valeur allant de 30 à $39^{\circ} \mathrm{C}$, la relation entre la température du dos (Y) et la moyenne sur 5 points $(\mathrm{X})$ est de la forme :

$$
\mathrm{Y}=0,976 \mathrm{X}+\mathrm{I}, \mathrm{I} 4 \quad r=0,90
$$

Cette droite est très proche de la droite $\mathrm{Y}=\mathrm{X}$, mais la dispersion des points est forte. Ceci est vraisemblablement dû aux variations instantanées de Y. L'utilisation de la température de surface du dos peut être envisagée pour des enregistrements, lorsque l'appareil ne peut être déplacé : on peut alors éliminer ces variations en utilisant des moyennes dans le temps.

\section{B. - Influence de la température et de l'humidité de l'air ambiant}

Il importe de vérifier leur homogénéité dans le local expérimental ou de faire plusieurs mesures psychrométriques au voisinage des différentes parties du corps dont on détermine $T_{s}$; on peut ainsi calculer les échanges thermiques locaux pour chaque partie, ainsi que la moyenne pour l'ensemble de l'animal.

\section{C. - Mesure de la température radiative des parois}

Dans un local quelconque, elle n'est pas homogène. L'utilisation d'un bilanmètre, qui intègre le rayonnement reçu sur un angle solide de $2 \pi$ stéradians, permet de résoudre ce problème, ainsi que celui posé par les mesures en plein air. Par contre, dans un local aux parois suffisamment isolantes, cette température s'homogénéise : on peut alors se contenter d'une mesure radiothermométrique.

\section{D. - Mesure de la résistance de la conche limite d'air au-dessus de la peau $\left(v_{a}\right)$}

On la détermine sur une peau

- qui n'est pas celle de l'animal étudié,

- qui est tendue sur un support plan,

- qui n'est pas forcément dans les mêmes conditions de ventilation que l'animal,

- qui du fait de sa température différente de celle de la peau de l'animal, n'est pas forcément soumise au même régime de convection. 
On peut améliorer la forme du support ; l'utilisation d'anémomètres à fil chaud permet de résoudre le problème de la ventilation. D'ailleurs, aux vitesses de vent faibles, $r_{a}$ semble pratiquement constant $\left(300 \mathrm{~s} \cdot 1 \mathrm{~m}^{-1}\right.$ et $270 \mathrm{~s} \cdot \mathrm{m}^{-1}$ respectivement pour deux peaux différentes, dans le local expérimental avec un écart-type de seulement ro $\mathrm{s} \cdot \mathrm{m}^{-1}$ malgré la ventilation très variable).

Il reste qu'on mesure la résistance pour une peau qui n'est pas celle de l'animal: on peut toutefois la choisir la plus semblable possible (en particulier pour la densité du pelage).

Comme la température de la peau isolée et mouillée est légèrement inférieure à celle de l'air ambiant, on est très certainement en présence d'un régime de convection forcée. Par contre, la peau des animaux rivants ayant, dans nos expériences, une température d'environ $\mathrm{I}^{\circ} \mathrm{C}$ supérieure à celle de l'air ambiant, on peut se demander si, alors, un phénomène de convection naturelle n'intervient pas, ce qui modifierait la valeur de $r_{a}$.

Montheith (I973) montre que, si

$$
\frac{\mathrm{Gr}}{\mathrm{Re}^{2}}<\mathrm{O}, \mathrm{I}
$$

$\mathrm{Gr}=$ nombre de Grashof (traduisant le rapport entre la poussée d'Archimède et la force de viscosité) ;

$\mathrm{Re}=$ nombre de Reynolds (traduisant le rapport entre les forces d'inertie et de viscosité).

on a un phénomène de convection forcée. Fn développant les expressions de ces detux nombres sans dimensions, l'équation (ro) se ramène à :

$$
\mathrm{V}>0,6 \sqrt{\mathrm{d} \overline{\Delta \mathrm{T}}}
$$

$\mathrm{V}$ : vitesse du flux d'air $\left(\mathrm{m} \cdot \mathrm{s}^{-1}\right)$

$\mathrm{d}$ : diamètre du corps des porcelets $(\mathrm{m})$

$\Delta \mathrm{T}$ : écart de température entre la peau et l'air ambiant $\left({ }^{\circ} \mathrm{C}\right)$.

Par exemple, si $d=0,3 \mathrm{~m}$ et $\Delta \mathrm{T}=\mathrm{I} 0^{\circ} \mathrm{C}$, il n'y a pas convection libre si $\mathrm{V}>\mathrm{I}$ $\mathrm{m} \cdot \mathrm{s}^{-1}$. En fait, la convection forcée peut être dominante à des vitesses de vent inférieures, par exemple pour l'homme nu (Mon'teITH, I973), lorsque $\Delta \mathrm{T}=\mathrm{I}^{\circ} \mathrm{C}$, si $\mathrm{V}>0,5 \mathrm{~m} \cdot \mathrm{s}^{-1}$. C'est sans doute le cas dans un local bien ventilé.

Par contre, dans un local fermé où la vitesse de l'air est de l'ordre de $0,2 \mathrm{~m} \cdot \mathrm{s}^{-1}$, la convection libre peut jouer un rôle déterminant. Seule l'expérience (par exemple sur une peau chauffée) peut permettre de déterminer le type de convection dans un environnement donné.

La convection forcée peut être le type laminaire ou turbulent. Le critère classique (nombre de Reynolds) ne permet pas, dans nos conditions expérimentales, de formuler une conclusion.

De toute façon, le calcul présenté ci-dessus (équation 7) reste valable en régime turbulent, au nombre de Lewis près $\left(\mathrm{L}_{\mathrm{e}} \mathrm{e}^{2 / 3}\right)$, ce qui introduit une erreur d'environ I 2 p. Ioo dans le calcul du flux de chaleur sensible, qui ne représente lui-même que $35 \mathrm{p}$. Ioo du dégagement total de chaleur. Si la peau isolée et la peau des animat1x présentent le même type de convection forcée, l'erreur est donc négligeable. 


\section{E. - Problème du fux de chaleur latente}

Comme nous l'avions indiqué, ce flux a été estimé à partir de mesures antérieures (cf. $\S I I, D)$.

\section{F. - Problème des pertes de chaleur par conduction}

Les porcelets en expérience ayant été élevés sur caillebotis, il ne s'est pas posé. Pour l'étudier, il faut connaître la conductivité du sol, le gradient de température produit par l'animal et la surface de contact pour différentes postures de ce dernier.

\section{G. - Résultats}

Malgré les imperfections de la méthode, les résultats que nous avons obtenus concordent avec les valeurs obtenues par d'autres auteurs, en particulier INGRAM (I964 $a$ et $b$ ) qui a effectué des mesures des échanges thermiques locaux à l'aide de fluxmètres et de capsules ventilées. En effet, nos résultats sont comparables aux siens pour la température de surface de la peau $\left(3^{\circ} \mathrm{C}\right.$ à une température ambiante de $28^{\circ} \mathrm{C}$, $33^{\circ} \mathrm{C}$ à $24^{\circ} \mathrm{C}$ ) et le coefficient de conduction du corps (de io à Ig $\mathrm{W} \cdot \mathrm{m}^{-2} \cdot{ }^{\circ} \mathrm{C}^{-1}$, valeurs qui, selon lui, correspondent à la vasoconstriction et à la vasodilatation maximales). Par contre, nos valeurs du flux de chaleur non évaporatif $\left(90 \mathrm{~W} \cdot \mathrm{m}^{-2}\right.$ à $2 \mathrm{I}^{\circ} \mathrm{C}$, $\left.75 \mathrm{~W} \cdot \mathrm{m}^{-2} \mathrm{à} 27^{\circ} \mathrm{C}\right)$ sont légèrement supérieures aux siennes $\left(8 \mathrm{o} \mathrm{W} \cdot \mathrm{m}^{-2}\right.$ et $6 \mathrm{o} \mathrm{W} \cdot \mathrm{m}^{-2}$ respectivement), avec il est vrai une température rectale plus élevée. Nous n'avons pas trouvé, sur le porcelet, d'autres résultats directement comparables aux nôtres, mais les nombreuses mesures calorimétriques des pertes de chaleurs globales donnent le même ordre de grandeur.

\section{V. - CONCLUSION}

Nous avons tenté, à la suite de l'exposé théorique de la méthode, d'énumérer les problèmes qu'elle pose, et qui seront d'autant plus importants que les conditions ambiantes sont plus variables et plus mal définies. Dans un environnement aussi bien défini que l'intérieur d'un calorimètre, la plupart des questions discutées cidessus ne se poseraient même pas ; mais alors cette méthode serait comparée à son désavantage aux méthodes calorimétriques directes ou indirectes. Son intérêt essentiel est d'être simple sur le plan pratique et d'être utilisable dans la plupart des conditions d'élevage, y compris, avec quelques modifications, l'élevage en plein air. Eille présente aussi l'avantage de mesures à distance, qui troublent moins les animaux. Sa précision dimintue avec la stabilité de l'ambiance : cependant elle est sans doute la seule à pouvoir être utilisée dans des conditions climatiques non contrôlées. 


\title{
SUMMARY
}

\author{
MEASUREMENT OF CUTANEOUS HEAT EXCHANGES \\ IN PIGS REARED UNDER SHELTER BY MEANS \\ OF THE ENERGY BAIANCE, METHOD
}

Measurement of local cutaneous heat exchanges was made by means of a method based on the physical principles pertaining to the energy balance of a surface. The basic measurement was that of the skin surface temperature made by infra-red radiothermometry. Ambient conditions of temperature, moisture, radiation and eventually air movement were determined as well as the rectal temperature of the pigs. The calculations indicate the different heat fluxes, cutaneous heat losses and tissues conduction. Various problems, often connected with the physical hypotheses are discussed. In spite of its imperfection, this method is probably the only one that can be used in non-controlled ambient conditions.

\section{RÉFÉRENCES BIBLIOGRAPHIQUES}

Crosf W. H., Mount L. E., Star'T I. B., 1971. The influence of environmental temperature and plane of nutrition on heat losses from groups of growing pigs. Anim. Prod., 13, 285-294.

Fincir V. A., I972. Thermoregulation and heat balance of the East African eland and hartebeest. Am. J. Physiol., 222, 1374-1379.

Findlay J. B., ig72. Climatologic data needed to specify climatic stress. World Rev. Anim. Prod., 8, 39-44.

Fulder M. F., Borke A.W., I972. The effects of environmental temperature on the growth and metabolism of pigs given different amounts of food. 2. Energy metabolism. Br. J. Nutr., 28, 37.3-384.

Holmes C. W., I 968 . Heat losses from young pigs at three environmental temperatures measured in a direct calorimeter. Anim. Prod., 10, I35-147.

Holmes C. W., 1973. The energy and protein metabolism of pigs growing at high ambient temperatures. Anim. Prod., 16, II7-133.

INGram D. L., I 964 a. The effect of Environmental Temperature on Heat Loss and Thermal Insulation in the Young Pig. Res. Vet. Sci., 5, 357-364.

INGRAM D. L., I964 b. The Effect of Environmental Temperature on Body Temperatures, Respiratory Frequency and Pulse Rate in the Young Pig. Res. Vet. Sci., 5, 348-356.

Incram D. L., I965. The Jiffect of Itumidity on Temperature Regulation and Cutaneous Water Loss in the Young Pig. Res. I'et. Sci., 6, 9-17.

KLliser M., I96I. The five of life. John Wiley and Sons, New York, London, 129-I44.

Montertil J. L., 1973. Principles of Environmental Physics, Edward Arnold Ltd, London, $24 \mathrm{I}$ p.

Morrison S. R., I972. Physical principles of energy exchange. J. Anim. Sci., 35, 624-627.

Molnt L. li., I968. The climatic Physiology of the Pig. Ed. Arnold Ltd, London, 27 I p.

Pirrier A., I970. Nesure des températures de surface par radiométrie infra-rouge. In Techniques d'étude des facteurs physiques de la biosphève, I. N. T. A., Paris, I69-I78.

PERrier A., I975. L'eau dans la Biosphìre. Tome I, Edit. Baillère (à paraître). 